\title{
Evaluation of a shortened course of tranexamic acid for patients with inherited bleeding
dental procedures
}

Allison Greig

People with Inherited Bleeding Disorders (IBD) are often prescribed a course of Tranexamic Acid (TXA) mouthwash for five to seven days following dental procedures to reduce the risk of bleeding. Informal discussions with patients suggested that many do not complete the prescribed course of treatment. A literature review indicated that TXA was prescribed inappropriately for procedures with a low bleeding risk, and that there are inconsistencies in the recommended dose, mode of administration and duration of TXA for this patient group. A new protocol was implemented in the haemophilia centre at St George's University Hospital NHS Foundation Trust, London, to rationalise the prescribing of TXA in dental procedures. A study was conducted to explore patients' experience of this new guideline in the form of a service evaluation. Structured telephone interviews were completed following 39 dental procedures to collect data on concerns about bleeding; whether TXA was taken as prescribed and reasons for non-adherence; and any unplanned post-operative treatment. The financial impact of the new guideline was also explored.

ALLISON GREIG

Clinical nurse specialist, Centre for Haemostasis and

Thrombosis - Haemophilia Centre, St George's University

Hospitals NHS Foundation Trust, London, UK. Email:

Allison.Greig@stgeorges.nhs.uk

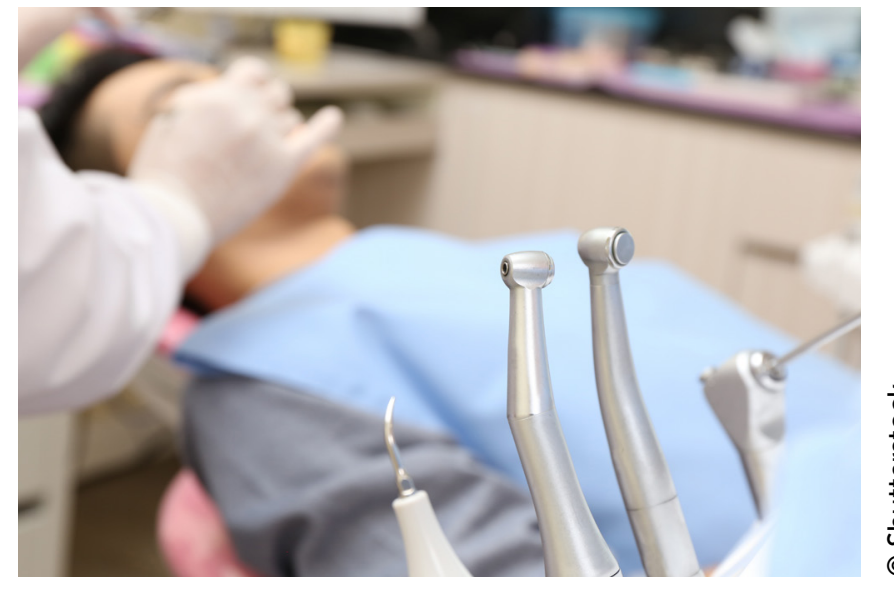

Patients were supportive of the new regimen, although almost half $(46 \%)$ did not complete the prescribed course of TXA. The majority (37/39) were prescribed tablets rather than mouthwash. No patients required additional unplanned haemostasis support to control haemorrhage. Cost savings were made by replacing a five- to seven-day course of TXA mouthwash with a three-day course of TXA tablets. Although the data collected from patient interviews supports the new guideline, patients appear to be making decisions about taking TXA based on their own experience rather than following the prescribed regimen. Prescribers should support patients to make informed decisions about their medicines and incorporate patient experience into individualised regimens. Given the lack of bleeding complications experienced in this

This is an Open Access article distributed under the terms of the Creative Commons Attribution-NonCommercial-NoDerivs License (https://creativecommons.org/licenses/by-nc-nd/3.0/) which permits use and distribution in any medium, provided the original work is properly cited, the use is non-commercial, and no modifications or adaptations are made. Copyright is retained by the authors. 
cohort of patients, it is possible that TXA is being overprescribed. Further work exploring how patients with IBDs make decisions about taking medicines is needed.

Keywords: Antifibrinolytics, Bleeding disorders, Dental procedures, Haemophilia, Tranexamic acid, Service evaluation

eople with inherited bleeding disorders (IBD) face increased risk of bleeding following invasive procedures unless haemostasis support is provided ${ }^{[1]}$. Clotting factor concentrate (CFC), desmopressin (DDAVP), platelet transfusion and/or antifibrinolytic therapy are typically used to support haemostasis during and after invasive procedures. Dental procedures are the most common invasive procedures performed on this population ${ }^{[2]}$. Close collaboration between patients, dental surgeons and haemophilia specialists is essential to reduce risk of bleeding complications ${ }^{[3]}$.

Tranexamic acid (TXA) is the most potent antifibrinolytic agent available. It is used to prevent surgical bleeding in orthopaedics, cardiothoracic surgery, obstetrics, gynaecology, neuro-surgery and dentistry, and has been found to reduce mortality following trauma, gastro-intestinal bleeding and head injury ${ }^{[4]}$. It is a cheap and versatile drug and is included on the World Health Organisation's list of essential medicines ${ }^{[5]}$.

In people with IBD it has been demonstrated that clot stability is reduced even after treatment with CFC, but that the addition of TXA improves both clot stability and efficacy of CFC ${ }^{[6]}$. Following dental surgery, the routine use of TXA has been shown to reduce the risk of delayed bleeding, clot breakdown and infection ${ }^{[7]}$. A high concentration of antifibrinolytic activity in saliva is also known to contribute to premature clot breakdown in the presence of an IBD ${ }^{[8]}$.

While antifibrinolytics have been used routinely to support haemostasis since the 1970 s $^{[4,9]}$, European and UK guidelines fail to provide specific recommendations with regard to the optimum mode of administration, dose, frequency and duration ${ }^{[10,11]}$. A Cochrane review in 2015 supported the use of TXA for patients with IBD undergoing dental procedures ${ }^{[2]}$; however, it was acknowledged that there was insufficient high-quality published evidence, such as that generated by a randomised controlled trial.

Following a review of prescribing practices in the haemophilia centre at St George's Hospital, London, in 2015, inconsistencies in prescribing TXA were identified. TXA in a mouthwash formulation was prescribed for

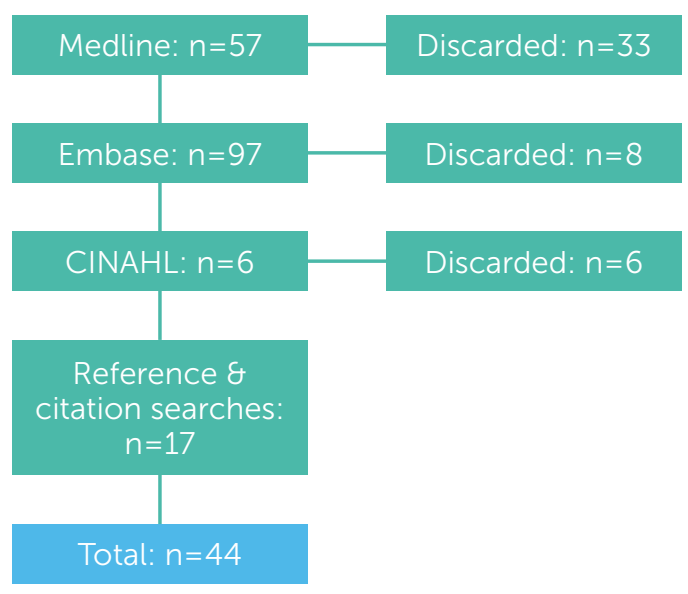

Figure 1: Literature search strategy

all patients with IBD undergoing dental procedures, regardless of the severity of bleeding disorder or bleeding risk associated with the procedure. Typically, a dose of $1 \mathrm{~g}$ $(20 \mathrm{ml})$, to be taken six hourly for five to seven days was prescribed. Anecdotal evidence from patients, suggested that many did not complete the course as prescribed and had not experienced any bleeding complications. At that time (2015), TXA mouthwash was prepared in the hospital pharmacy and dispensed in $300 \mathrm{ml}$ bottles (concentration $500 \mathrm{mg} / 10 \mathrm{ml}$ ) at a cost of $€ 145$ each; two bottles are required for five to seven days' treatment. In January 2017, during the course of the data collection period for this study, the hospital started to buy in TXA mouthwash from an external supplier at a cost of $€ 32.58$ per $100 \mathrm{ml}$ (concentration $500 \mathrm{mg} / 5 \mathrm{ml}$ ).

A review of published literature was undertaken in February 2016, using the Medline, Embase and CINAHL healthcare databases. The following search terms were used in combination: 'tranexamic acid', 'cyclocapron', antifibrinolyt*', 'dental surgery', 'dentoalveolar', 'dental extraction', 'molar', 'haemophilia', 'hemophilia', 'Willebrand', 'bleeding disorder'. A summary of the literature search strategy is shown in figure 1.

The literature review revealed wide inconsistencies in the use of TXA as a mouthwash and/or tablets along with variations in the prescribed dose with regimens of six to eight hourly, for three to 14 days. No conclusive evidence was found to advocate the efficacy of mouthwash compared with tablets, although TXA tablets are significantly cheaper than mouthwash ${ }^{122]}$. Anderson et al. suggest that tablets can be crushed by the patient and added to $10 \mathrm{ml}$ water to produce an oral solution that can be used as a mouthwash at a lower cost ${ }^{[10]}$, and the use of an oral solution prepared by patients using crushed tablets is reported by Nuvvula et al. ${ }^{[13]}$. This method of administration is outside of 


\begin{tabular}{|c|c|}
\hline All patients & $\begin{array}{l}\text { - TXA to be commenced two hours before dental procedure } \\
\text { - TXA to be taken every six hours for three days post-procedure }\end{array}$ \\
\hline Moderate or severe IBD & $\begin{array}{l}\text { - TXA mouthwash solution - to be swished around the mouth for two minutes, then } \\
\text { swallowed }\end{array}$ \\
\hline Mild IBD & $\begin{array}{l}\text { - TXA tablets, OR } \\
\text { - If postoperative bleeding is noted, TXA tablets crushed, mixed with water and used as a } \\
\text { mouth rinse for two minutes, then swallowed }\end{array}$ \\
\hline
\end{tabular}

Table 1: Amended TXA prescribing guidelines for patients with IBDs undergoing dental procedures

\section{Diagnosis of Respondents}

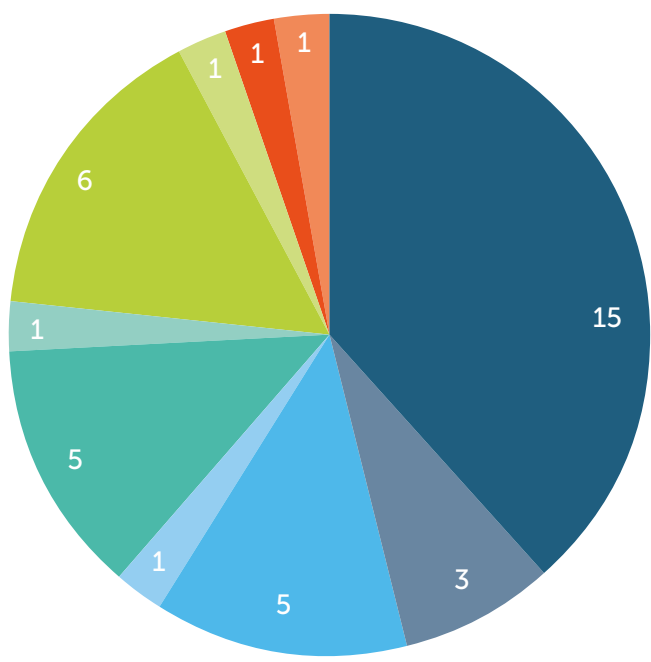

Von Willebrand's disease

Platelet function disorder

Unclassified

Mild haemophilia A

Glanzman's thrombasthenia

Figure 2: IBD diagnosis of patients interviewed

the product licence and no efficacy data is available; however, anecdotal evidence from colleagues in other London haemophilia centres supports this practice. This method would result in significant cost saving compared to the pharmacy-dispensed mouthwash; but may be less convenient for patients.

At St George's Hospital, it was established practice to advise patients to swish TXA mouthwash gently around the socket or injection site, then swallow; however, no recommendation with regard to how long the solution should be swished in the mouth was given either by staff or on the product label and information sheet. UKHCDO guidance suggests that the TXA solution should be gently swilled inside the mouth for two to three minutes and then swallowed or gently expelled ${ }^{[10]}$. Anderson et al. also note that the use of TXA as a mouthwash provides antifibrinolytic activity in saliva, which is not found when taken as a tablet ${ }^{[10]}$. Therefore, a combination of local (mouthwash) and systemic (tablets) TXA may be more effective than monotherapy or swallowing of mouthwash rather than expelling. This recommendation is supported by SindetPedersen and Stenbjerg ${ }^{[8]}$.

Following a review of available evidence and guidelines, the haemophilia clinical nurse specialists and haemostasis consultants decided together that the local prescribing protocol should be amended (see table 1). It was decided to offer TXA mouthwash solution only to patients with moderate or severe disorders, who were thought to be at the greatest risk of bleeding. Those with mild bleeding disorders would be prescribed TXA tablets; if postoperative bleeding was noted, they would be advised to crush the tablets and mix them with water, then use the solution as a mouth rinse for two minutes before swallowing. TXA was to be commenced two hours before the procedure and to be taken every six hours for three days.

It was anticipated that some patients would have concerns with regard to the change in practice and that evidence would be needed to document that patients were not at increased risk of bleeding. Therefore, a service evaluation was undertaken to explore patients experience of the newly implemented guideline. The objectives were:

- To establish whether patients had concerns about bleeding after a dental procedure

- To determine whether the TXA was taken as prescribed

- To investigate whether any unplanned treatment or interventions were required

- To document patients' comments on their treatment

- To compare outcomes between mouthwash and tablets

- To identify whether cost savings could be made.

\section{METHOD}

This project was undertaken as a service evaluation, which is defined by the NHS Health Research Authority as an activity used to define or judge current care without reference to a standard ${ }^{[14]}$. A draft interview 


\section{TRANEXAMIC ACID: INFORMATION FOR PATIENTS \\ You have been prescribed Tranexamic Acid either as a mouthwash or tablets to reduce bleeding following a dental procedure.}

If using mouthwash, this should be gently swished around the mouth for 2 minutes and then swallowed 4 times a day for 3 days starting on the day of the procedure.

Tablets should be taken 4 times a day for 3 days starting on the day of the procedure.

A haemophilia nurse will telephone you within a few weeks of the procedure to find out if you had any concerns or difficulties with your treatment.

Alternatively, please feel free to contact the haemophilia nurses on 02087250763

The questions you will be asked are:

Did you have any concerns about the amount of bleeding in the days following your dental procedure? Yes/No

Did you need any additional treatment to reduce blood loss after the procedure? Yes/No

If yes; what treatment did you receive?

How many days did you take Tranexamic Acid?

If Tranexamic Acid was taken for more or less than 3 days, why was this?

Do you have any other comments?

MANY THANKS FOR YOUR HELP: YOUR ANSWERS WILL BE STRICTLY CONFIDENTIAL YOU HAVE THE RIGHT NOT TO TAKE PART IN THE SURVEY IF YOU WISH

schedule was developed with the help of colleagues, based on previously published tools but adapted to meet the needs of this study. As recommended by McColl ${ }^{[15]}$, and with further help from colleagues, the tool was tested and revised until the researcher was confident that it was fit for purpose.

The following criteria was used to identify patients eligible to be included in the study:

Inclusion criteria:

- Registered with the adult haemophilia team

- Diagnosed with an IBD

- Undergoing a dental procedure likely to provoke bleeding. Assessment of bleeding risk based on UKHCDO guidelines ${ }^{[10]}$ and the judgment of the dental surgeon/hygienist.

\section{Exclusion criteria:}

- TXA contra-indicated due to medical history/comorbidities

- Non-English speakers
The researcher was mindful that excluding non-English speakers may introduce an element of bias into the sample. However, the number of participants excluded was recorded, and the possible impact on results discussed in the light of the study findings.

An information sheet explaining the purpose of the study and the questions to be asked was handed or posted to patients by the researcher prior to their dental procedure (see figure 2) ${ }^{[16,17]}$. Patients were advised to contact the haemophilia team if they had any questions or concerns.

Telephone interviews were used to collect data between April 2016 and September 2017. Based on the structured interview schedule, the aim was to obtain quantitative data that was unambiguous and easy to interpret. The opportunity to give any additional comments at the end of the interview was included to give interviewees the opportunity to expand on their experience and give context to their responses.

Respondents' comments were documented verbatim and subsequently quantified into categories. 
Table 2: Summary of patients referred for dental procedures during the study period

\begin{tabular}{l|l|l|l|l}
\hline PROCEDURE & INTERVIEWED & EXCLUDED & NON-RESPONDER & TOTAL \\
\hline Extraction & 30 & 2 & 8 & 40 \\
\hline Hygienist & 6 & 4 & 4 & 14 \\
\hline Oral surgery & 3 & 0 & 0 & 3 \\
\hline All & 39 & 6 & 12 & 57 \\
\hline
\end{tabular}

Frequency tables were used to describe the sample demographics, diagnosis and procedure undertaken. Cross-tabulation tables were used to compare variables across patient groups. It was intended that either a chi-squared test or Fisher's exact test would be used to compare outcomes between the use of TXA mouthwash and tablets and also between different patient groups.

Interpretations from the data were reviewed with more experienced researchers within the haemophilia team and the project supervisor.

\section{RESULTS}

During the data collection period, 50 patients with IBD were referred to the haemophilia team for management of haemostasis support for 57 dental procedures. All patients were prescribed TXA. Thirty-nine telephone interviews were carried out with 34 patients; five patients had two separate procedures and therefore participated in two interviews. Five patients (having six procedures) were excluded, all of whom were non-English speakers. Eleven patients (undergoing 12 procedures) chose not to take part in the interviews. Table 2 summarises the total number of patients referred for dental procedures during the study period, including those who were excluded from or did not participate in the interviews. The IBD diagnosis of the patients interviewed is shown in figure 2.

All patients were prescribed TXA 1g, either as a tablet or mouthwash, to be taken four times daily for three days following their dental procedure. One patient had a reduced dose (1.5g, twice daily) due to renal insufficiency. The procedures undertaken, along with the patients' age and length of treatment is shown in table 3 .
Patients, who took TXA for longer than three days, were older (aged 52-81 years); however, there were only four patients in this group. There was no difference in mean age for those who took TXA for three days or less. A chi-squared test was used to compare duration of treatment between males and females, and no statistically significant difference was found $(p=0.79)$.

Patients were asked to give a reason for over- or under-use of TXA. Sixteen out of 18 patients (88\%) who did not complete the course said that they did not feel that longer treatment was warranted as they did not experience any bleeding. Of the four patients that used TXA for more than three days, three gave the following reasons:

- "I lost count of the days"

- "I had an infection and decided to treat for an extra day"

- "I reduced the frequency of TXA to once daily due to nausea but continued for an extra day."

The fourth patient was given TXA tablets for three days as per the protocol, but then travelled to a local hospital for the procedure (extraction). The local hospital gave the patient a prescription for seven days of TXA mouthwash, which is what she took.

Only six patients used TXA as a mouthwash, of which three were interviewed (one was excluded as a non-English speaker, and two declined to take part). As data were lacking, it was not possible to make any comparisons between TXA tablets and mouthwash with regard to effectiveness or patient adherence.

The majority of patients requiring haemostasis support were prescribed TXA as a single therapy (25/39). CFC or DDAVP was administered before the dental procedure in 14 cases. One patient required an additional dose of CFC on the first post-operative day for facial swelling. There were no reports of

Table 3: Treatment adherence in relation to age and procedure

\begin{tabular}{|c|c|c|c|c|}
\hline VARIABLES & TXA <3 DAYS & TXA 3 DAYS & TXA >3 DAYS & TOTAL \\
\hline Age (mean) & 50 years & 50 years & 75 years & 51 years \\
\hline Extraction & 10 & 16 & 4 & 30 \\
\hline Hygienist & 6 & 0 & 0 & 6 \\
\hline Oral surgery & 2 & 1 & 0 & 3 \\
\hline
\end{tabular}




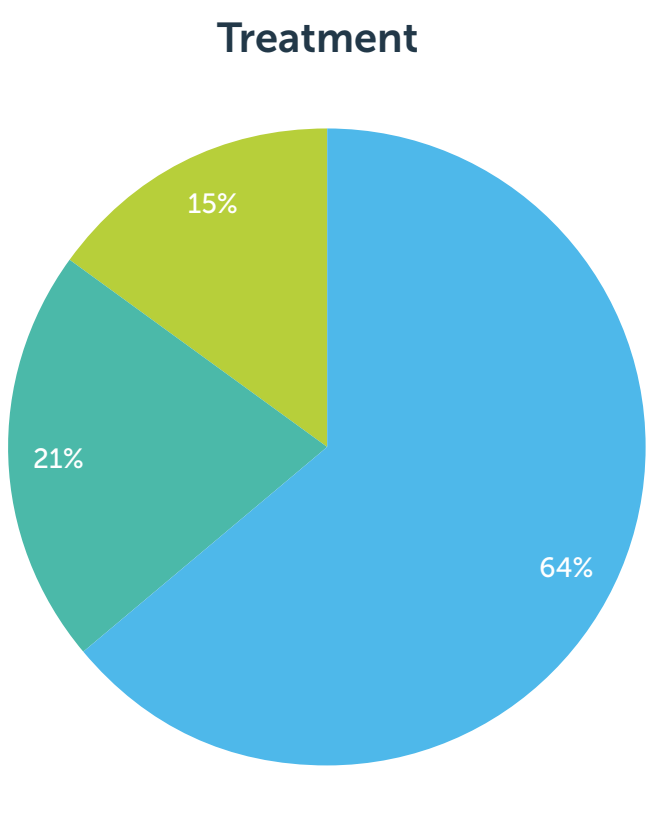

TXA only

CFC \& TXA

DDAVP \& TXA

Figure 4: Haemostasis treatment prescribed for dental procedures

haemorrhage. Six patients needed to return to the dentist or GP for further assessment post-procedure, four of whom required antibiotics. Figure 2 shows the haemostasis treatments prescribed.

Based on the number of procedures performed during the study period, an estimate of the costs of the newly implemented guideline was compared to the previous regimen. Prior to February 2016, patients undergoing dental procedures were usually prescribed TXA mouthwash at a cost of $£ 290$ for five to seven days. Following this protocol would have cost $£ 16,530$. Five patients were prescribed TXA mouthwash during this service evaluation; however, only three days of treatment were prescribed. The cost was £145 per procedure for four patients who had procedures prior to January 2017 (when the supplier of TXA mouthwash changed), and $£ 32.58$ for one patient who had a procedure after January 2017. Three patients were prescribed both mouthwash and tablets. TXA tablets are dispensed in boxes of 60; the five patients who had two procedures would have required only one box for both procedures. Table 4 shows that the total cost of TXA treatment was $£ 815.14$, producing a saving of over £15,700.

Overall, analysis of the data collected demonstrates the following:

- There were no incidents of excessive bleeding following the implementation of the new protocol

- Patient feedback suggests that duration of TXA could be reduced further in some cases

- Patients made their own decisions about taking TXA based on their symptoms

- $43 \%$ of patients completed the course of TXA as prescribed

- Significant cost savings were made

\section{DISCUSSION}

Published studies and guidelines recommend TXA is taken three or four times daily for two to ten days; the majority of authors recommend five to seven days. However, other than in the setting of a randomised controlled trial, patient compliance with these recommendations was assumed. This service evaluation revealed that a shortened course of TXA provided effective haemostasis support, as the vast majority of procedures (38/39) did not warrant any additional haemostasis treatment.

One patient required an additional unplanned dose of CFC, following extraction of four wisdom teeth. She was admitted to the hospital for observation after her procedure due to the high bleeding risk. Although the patient did not have a haemorrhage, further CFC was given due to the amount of facial swelling 24 hours after the extractions. When the patient was seen by the maxillofacial team, they were not concerned about the amount of swelling and were happy for her to be discharged. The patient completed three days of TXA as prescribed and did not have any concerns about bleeding after discharge.

Telephone interviews were not undertaken after 18 procedures, either because patients did not respond to the interview invitation or did not meet the inclusion criteria. However, the haemophilia team did not receive any communication from these patients or

Table 4: Costs of TXA prescribed during the study period

\begin{tabular}{|c|c|c|c|}
\hline FORM OF TXA PRESCRIBED & NO. OF PATIENTS & $\operatorname{COST}(\mathrm{f})$ & TOTAL (f) \\
\hline Tablets only & 45 & 4.22 & 189.90 \\
\hline Mouthwash only & 2 & 145.00 & 290.00 \\
\hline Tablets and mouthwash & 3 & 149.22 & 447.66 \\
\hline Total & 50 & & 927.56 \\
\hline
\end{tabular}


the dentist with regard to any complications or for further treatment and/or assessment of post procedure bleeding. It can therefore be surmised that a shortened course of TXA was also effective in this group.

It was intended that patients with moderate or severe bleeding disorders would be prescribed TXA as a mouthwash. This decision was made on the basis that the mouthwash was likely to be more effective by providing both local and systemic antifibrinolytic activity when swished and swallowed. The results reveal that five patients were prescribed mouthwash, with one further patient being prescribed tablets but using mouthwash prescribed at another hospital. Three patients were prescribed mouthwash in combination with tablets, none of whom met the prescribing criteria for mouthwash. Where a decision was made to use mouthwash, this was generally pragmatic and based on the risk of bleeding associated with the dental procedure rather than the patient's bleeding disorder diagnosis. During the study period the practice of using both mouthwash and tablets emerged as a pragmatic solution to providing antifibrinolytic activity locally while reducing the volume and costs associated with mouthwash only. In these cases $500 \mathrm{mg}$ in $10 \mathrm{ml}$ of mouthwash was prescribed to be swished around the mouth for two minutes and swallowed along with a $500 \mathrm{mg}$ tablet taken at the same time.

Thirty patients were interviewed following extraction procedures. This group were the most likely to be adherent or over-treat (20/30;67\%) compared to those having oral surgery or a hygienist procedure (1/9; $11 \%)$. All six patients who had a hygienist procedure took their TXA for less than three days and reported lack of bleeding as the reason why they discontinued the treatment. One claimed that they would be happy to have the procedure without any TXA next time. Another, who took TXA for two days, did not report any bleeding but felt that the TXA gave him "psychological reassurance that I won't bleed". Certainly, the UK guidelines suggest that haemostasis support should only be required for hygienist interventions if the general health of the gums is very poor ${ }^{[10]}$.

The dental referral forms ask the hygienists to make a prediction of bleeding risk, which the haemophilia team then use to determine what treatment is required. Only those who were deemed to be at risk of bleeding were given TXA and included in this study. However, to accurately predict the risk of bleeding can be difficult and additional haemostasis support may not have been necessary for some of these patients. An option for future hygienist procedures would be to provide the patient with TXA tablets but let them and the hygienist make a decision on whether or not it is required based on the amount of bleeding during and after the procedure. Two studies comparing the use of TXA with placebo during dental scaling in people with haemophilia reported that $38.5 \%$ and $36 \%$ of patients respectively did not bleed, regardless of whether TXA was given or a placebo ${ }^{[13,18]}$. The majority of patients in these studies had severe haemophilia, while the six patients interviewed for the current study all had mild bleeding disorders. On reflection, treatment with TXA may be necessary only for hygienist procedures where there is poor gingival health alongside a severe bleeding disorder.

Of the four patients who took TXA for longer than three days, none did so in response to concerns about excessive bleeding. Following this study, the prescribing protocol has been further rationalised, with patients prescribed TXA 1g (either tablets or tablets and mouthwash in combination) to be taken six to eight hourly, for up to three days, according to symptoms.

\section{CONCLUSION}

This study demonstrated that patients did not experience excessive bleeding following the change in protocol around the use of TXA for dental procedures in patients with IBD, and that no additional unplanned haemostasis support was necessary. Feedback from patients suggests that many believe that TXA is not always necessary following dental procedures, as almost half $(46 \%)$ of the patients interviewed did not complete the three-day course of TXA. These patients had made a pragmatic decision to stop treatment that they believed was unnecessary. This raises the question of whether bleeding risk in some patients has been over-estimated. Improved access to CFC and desmopressin, along with better dental hygiene and dental techniques may have reduced bleeding risk over time.

The study also reveals patients making their own decisions about taking prescribed medication based on their symptoms. Engaging patients in guideline development and prescribing decisions has resulted in improved medicines optimisation and produced significant financial savings. Further exploration of how patients make decisions about taking prescribed medication is warranted.

\section{ACKNOWLEDGEMENTS}

The author has advised no interests that might be perceived as posing a conflict or bias.

The author would like to thank Dr Alan Vale, Senior Lecturer in Pharmacology, for his help in the writing of 
this project; Dr Steve Austin, Consultant Haematologist, and Anne Wareing, Haemophilia Nurse Specialist, for their help with designing the patient information sheet and interview schedule; Anne Wareing, Jacqueline Braithwaite-Smith and Julia Sexton, Haemophilia Nurse Specialists, for proofreading the original study manuscript; Dr James Uprichard, Consultant Haematologist, for his support throughout this study; and all the patients who took part.

This article reports on an evaluation study to which participants responded knowing any comments may be reported.

\section{REFERENCES}

1. Rasaratnam L. Risk-based management of dental procedures in patients with inherited bleeding disorders: development of a Dental Bleeding Risk Assessment and Treatment Tool (DeBRATT). Haemophilia 2017; 23(2): 247-54.

2. Van Galen KP, Engelen ET, Mauser-Bunschoten EP, van Es RJ, Schutgens RE. Antifibrinolytic therapy for preventing oral bleeding in patients with a haemophilia or von Willebrand disease undergoing oral or dental procedures. Cochrane Database Syst Rev 2015; (12): CD0011385. doi: 10.1002/ 14651858.CD011385.pub2.

3. Khyati C, Triveni MG, Gopal R, et al. Dental considerations in a patient with haemophilia. J Haem Pract 2016; 3(1): 51-4. doi:10.17225/jhp00070

4. Badle $S$, Hart D. Anti-fibrinolytic agents in bleeding disorders - a clinical perspective. J Haem Pract 2016; 3(2): 1-4. doi 10.17225/jhp00089.

5. Lecker I, Wang DS, Whissell PD, et al. Tranexamic acidassociated seizures: causes and treatment. Ann Neurol 2016; 79(1):18-26. doi: 10.1002/ana.24558.

6. Rea $\mathrm{CH}$, Foley JH, Beva DH, Sørensen B. An in-vitro assessment of tranexamic acid as an adjunct to $\mathrm{rFVIII}$ or $\mathrm{rFVIIa}$ treatment in haemophilia A. Ann Hematol 2014; 93(4): 683-92.
7. Tavenner RWH. Use of tranexamic acid in control of haemorrhage after extraction of teeth in haemophilia and Christmas disease. Br Med J 1972; 2(5809):314-5.

8. Sindet-Pedersen A, Stenbjerg S. Effect of local antifibrinolytic treatment with tranexamic acid in hemophiliacs undergoing oral surgery. J Oral Maxillofac Surg 1986; 44(9): 703-7.

9. Tengborn L, Blombäck M, Berntop E. Tranexamic acid - an old drug still going strong and making a revival. Thromb Res 2015; 135(2): 231-42. doi: 10.1016/j.thromres.2014.11.012.

10. Anderson JAM, Brewer A, Creagh D. Guidance on the dental management of patients with haemophilia and congenital bleeding disorders. Br Dent J 2013; 215(10): 497-504. doi: 10.1038/sj.bdj.2013.1097.

11. Hermans, C, Altisent C, Batorova A, et al; European Haemophilia Therapy Standardisation Board. Replacement therapy for invasive procedures in patients with haemophilia: literature review, European survey and recommendations. Haemophilia 2009; 15(3): 639-58.

12. British National Formulary, 2017. Available from http://www. medicinescomplete.com (accessed: 9 May 2017).

13. Nuvvula S, Gaddam KR, Kamatham R. Efficacy of tranexamic acid mouthwash as an alternative for factor replacement in gingival bleeding during dental scaling in cases of hemophilia: a randomized clinical trial. Contemp Clin Dent 2014; 5(1): 4953. doi 10.4103/0976-237X.128663.

14. NHS Health Research Authority. Defining research, 2013. Available from: www.hra.nhs.uk/documents/2013/09/ defining-research.pdf (accessed 25 February 2016).

15. Mccoll E. Questionnaire design and construction. Nurse Researcher 1993; 1(2): 16-25. doi: 10.7748/nr.1.2.16.s3.

16. Moule P, Goodman M. Nursing Research: An Introduction, 2nd ed. 2014. London: Sage.

17. Robson C. Real World Research, 3rd ed. 2011. London: Blackwell.

18. Lee APH, Boyle CA, Savidge GF, Fiske J. Effectiveness in controlling haemorrhage after dental scaling in people with haemophilia by using tranexamic acid mouthwash. Br Dent $\mathrm{J}$ 2005; 198(1): 33-8.

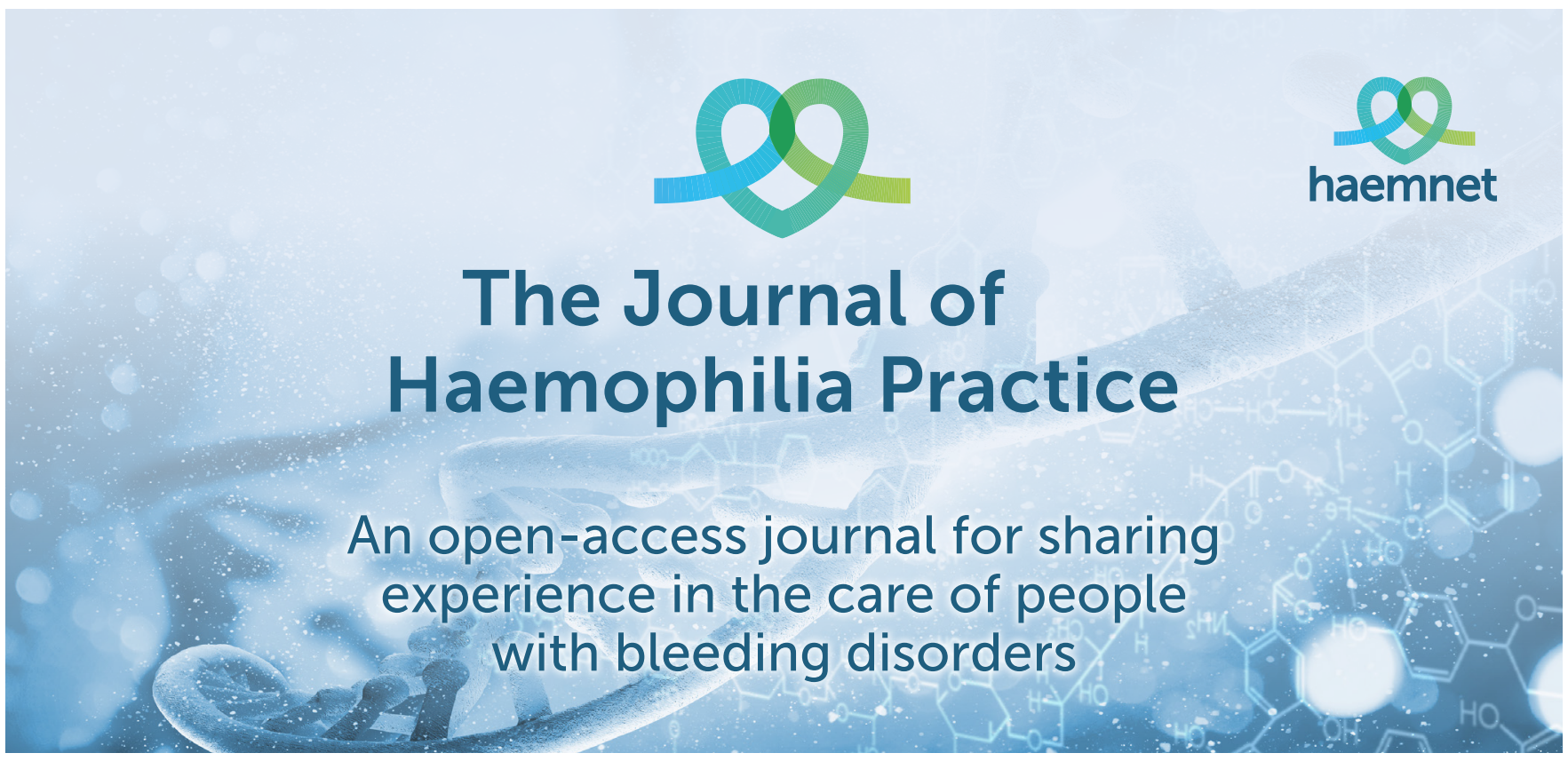

